\title{
Images in Anesthesia: Headache caused by pneu- mocephalus following inadvertent dural puncture during epidural space identification: is it time to abandon the loss of resistance to air technique?
}

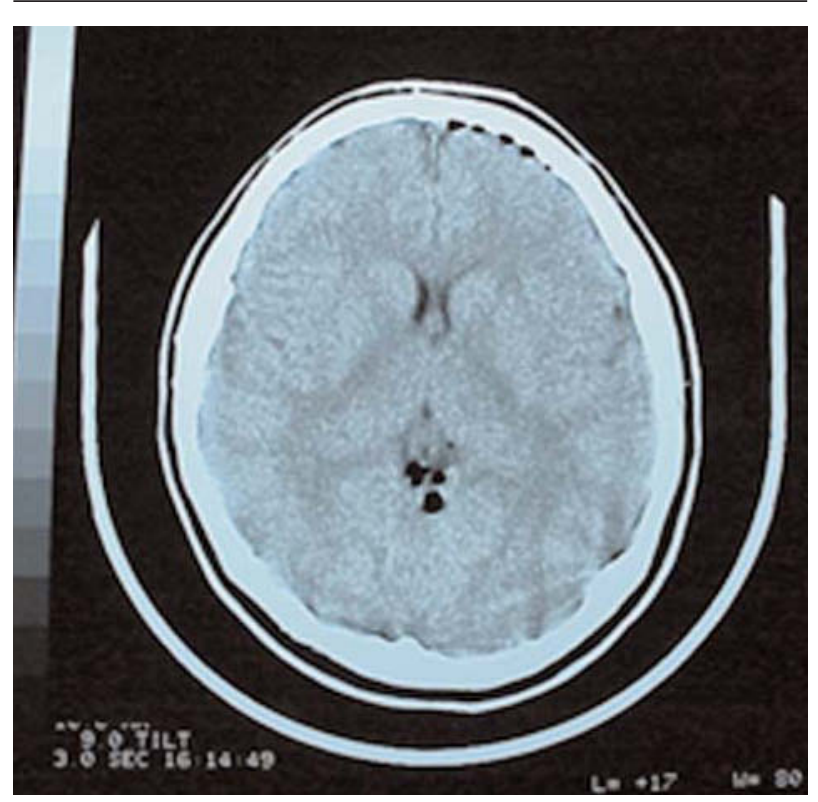

Computerized tomography of the cranium. Air is visible within the basilar cisterns and subarachnoid space anteriorly and along the falx over the convexities of the brain.

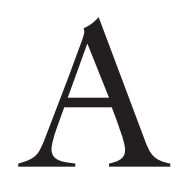

17-yr-old healthy female at 39 weeks gestation was in labour and requested labour analgesia. During the advancement of the 18-GA Tuohy-Schliff epidural needle at the L3-4 interspace with loss of resistance to air technique (LORA) an inadvertent dural puncture occurred and approximately $4 \mathrm{~mL}$ of air in the syringe was injected into the subarachniod space one second before the free flow of cerebral spinal fluid into the syringe established the diagnosis of dural puncture. The patient reported an immediate onset of a severe occipital headache. A second successful epidural attempt was made at the same interspace, this time with the loss of resistance to saline technique. The epidural block provided good pain relief for labour and vaginal delivery. However, the severe occipital headache continued unabated. Neurological examination revealed no deficits.
Computed tomography brain scan demonstrated the presence of intracranial air (Figure). The headache was treated conservatively and resolved in seven days. The LORA technique has been linked to complications including air embolism, compression of the cauda equina, increased incidence of dural puncture and pneumocephalus and has been criticized by some. ${ }^{1-5}$ This case adds one more piece of evidence that the safety of LORA technique should be reconsidered.

\section{Krzysztof M. Kuczkowski MD}

Jonathan L. Benumof MD

San Diego, California

\section{References}

1 Saberski LR, Kondamuri S, Osinubi Or. Identification of the epidural space: is loss of resistance to air a safe 
technique? A review of the complications related to the use of air. Reg Anesth 1997; 22: 3-15.

2 Laviola S, Kirvela M, Spoto MR, Tschuor S, Alon E. Pneumocephalus with intense headache and unilateral pupillary dilatation after accidental dural puncture during epidural anesthesia for cesarean section. Anesth Analg 1999; 88: 582-3.

3 Yentis SM. Time to abandon loss of resistance to air. Anaesthesia 1997; 52: 184.

4 Gonzalez-Carasco FJ, Aquilar JL, Llubia C, Nogues S, Vidal-Lopez F. Pneumocephalus after accidental dural puncture during epidural anesthesia. Reg Anesth 1993; 18: 193-5.

5 Aida S. Taga K, Yamakura T, Endoh H, Shimoji K. Headache after attempted epidural block: the role of intrathecal air. Anesthesiology 1998; 88: 76-81. 